\title{
APPLYING WEB USABILITY CRITERIA FOR VISION-IMPAIRED USERS: DOES IT REALLY IMPROVE TASK PERFORMANCE?
}

\author{
Barbara Leporini, Fabio Paternò \\ 56124 - Pisa, Italy \\ ISTI - C.N.R. \\ \{barbara.leporini, fabio.paterno\}@isti.cnr.it
}

\begin{abstract}
Available accessibility guidelines do not necessarily guarantee usable web sites, in particular when specific groups of users with special needs are considered. We have identified $15 \mathrm{Web}$ design criteria aiming to provide integrated support of accessibility and usability for vision-impaired users. In this paper, we present the results of a study investigating whether the application of such guidelines for vision-impaired users can actually improve their task performance when accessing Web applications. We report on two user tests, both involving vision-impaired users, which aim to provide empirical validation of the design criteria. During each test, users had to access and navigate two versions of a Web site: one version supporting the selected design criteria and one obtained with traditional techniques. Our results indicate that the 15 design criteria improved Web site usability both quantitatively and qualitatively by reducing the navigation time needed to perform the assigned tasks and by making the Web sites easier to navigate for blind and low-vision users.
\end{abstract}

\section{INTRODUCTION}

In recent years, the interest in accessibility and usability issues has increased. In fact, the use of Web sites has been widening, and the number of users accessing them is steadily increasing. For this reason, it is important that the information be easily reachable by all, including people with disabilities. Moreover, for some user categories (e.g. blind users) the Web is one of their main sources of useful information about educational or cultural purposes. The difficulties in providing such universal access can be addressed through the application of usability and accessibility principles. Accessibility is aimed specifically at making Web sites more available to a wider population of users (including special categories), by removing the technical barriers that prevent access to the information included in the site. To this end a number of initiatives at governmental level (such as the Section 508) and standardization level (such as the W3C WAI) have been undertaken to stimulate awareness of such issues in Web interface developers and service providers. Both W3C guidelines and section 508 standards consider mainly accessibility issues in order to define the general principles to be followed for removing technical barriers that might prevent the access to information. Thus, the main goal considered is the information access, whereas navigation easiness or simplicity of use is not specifically addressed.

However, accessibility alone is not enough. Usability aspects need to be addressed as well. Indeed, accessibility and usability are frequently addressed as two separate issues but disabled users need to have both accessible and usable applications. In fact, accessibility and usability are two intertwined aspects of Web site interaction and if they are not properly integrated, Web sites can turn out to be either accessible but barely usable, or usable but barely accessible. In both cases 
Web site navigation by disabled users is likely to be seriously compromised, since they either may not be able to access the desired information (i.e., it is not accessible) or may find difficulty in arriving at what they need (in this case it is not usable).

Our research has focused on the navigational problems encountered by blind/low-vision users. To this end, we have identified a set of criteria for design and evaluation of Web sites in order to make online services easier to use for vision-impaired users, who navigate through screen readers or magnifying programs. In order to bridge the gap between visual layout and aural perception, we have identified a specific set of criteria for improving interaction when using special devices (i.e. screen reader and magnifiers). In section 2.3 a short description of each criterion identified is provided for the reader's convenience. The goal of this paper is to assess the impact of the criteria identified in our previous work (Leporini and Paternò, 2004) on end users. In particular, we discuss the possible effects of the identified criteria by reporting the results of two tests carried out with forty visually-impaired users for an empirical validation of such criteria. Thus, we analyse the benefits obtained by visually-impaired people when navigating Web sites designed applying the identified usability criteria. We also present the results obtained by a questionnaire proposed to the users, regarding the qualitative aspects of navigation.

Usability testing provides an evaluator with direct information regarding the way people use applications and the problems they encounter when they use the tested interface. In our case, the two tests were conducted on blind and visionimpaired people who navigate through a screen reader. They were performed using two Web sites and for each test the subjects were assigned seven tasks. For each Web site two versions were developed: one by applying the test criteria (Implemented site) and the other without considering the criteria (Control site). The testing procedure adopted was the same for both tests and was based on two remote evaluation techniques (client-side automatic logging complemented with a remote questionnaire). Our tests revealed that when our criteria are applied, Web navigability is improved in terms of time spent looking for information or performing a task. Such empirical results provide interesting feedback on the impact of the application of the selected criteria on final users.

\section{BACKGROUND}

\subsection{NAVIGATING THROUGH ASSISTIVE TECHNOLOGY: VISUAL PERCEPTION VS. SCREEN READER INTERACTION}

Most blind people use screen readers with voice synthesizers rather than Braille displays for various reasons, amongst others, the difficulty of reading Braille for people who lost sight in an adult age. While low-vision users can interact by means of magnifying programs or simply through the operating system or single application's "easy access" settings (e.g. dynamic font resizing, colour enhancement, etc.). By using all these assistive technologies, the perception of the Web pages differs greatly from the usual navigation without special devices. In previous work (Leporini, 2003; Leporini and Paternò, 2004), we examined how visually-impaired users interact with a computer and how they perceive the content rendered. For the reader's convenience we have included a summary of the main issues. 
Screen readers convert vocally all the information in the Web page through a linear scan from top to bottom and from left to right. They also provide some support to access specific elements in the Web pages. Thus, it is useful a certain expertise in screen readers and browser commands, and orientating oneself within the page content can require considerable effort. Blind users usually do not use the mouse functions (i.e. pointing, scrolling, selecting, etc.) for moving around the page. They prefer moving by means of keyboard commands, such as the Tab key, arrow keys, and so on. Indeed, through specific screen reader commands mouse functionalities can be completely emulated by keyboard access, which allows moving the mouse pointer without having to use a physical mouse. For example, with the Jaws screen reader (Jaws, 2005) the mouse pointer - called the jaws cursor - can be controlled by the num-pad keys. In particular, the Jaws cursor (i.e., mouse emulator) is used for exploring the user interface or for reaching all those UI components that cannot receive the focus, such as tool bars or interactive elements, and can be activated only by a mouse click. However, a user interface with some interactive elements that cannot be activated through the keyboard (or that cannot gain the focus) is not usable. In fact, although the mouse can be emulated through the keyboard, all such movements require a great deal of effort by a blind user. Thus, developers should take into account all these considerations and build user interfaces that are fully accessible through the keyboard.

Even when Web sites apply accessibility recommendations, users can still encounter navigational problems. This is partly due to the fact that Web pages are increasingly designed for parallel or non-serial navigation, offering a variety of options within one page (frames, tables, drop down menus etc). Complex Web pages can cause problems for users who are navigating the site using assistive technologies, which force them to follow a serial route, for example a screen reader reading out each hypertext link on a page one by one.

Our approach is aimed at focusing on the main difficulties encountered by the visually-impaired when they interact with Web sites through some assistive technology. Then, starting with the main navigational problems for the blind and low-vision users, we investigated a possible solution by identifying several general design principles. In brief, the main problems for a blind person navigating through screen reader or magnifier are:

- Lack of page context - the user may loose the overall context of the current page when navigating through screen reader/magnifier, since they can read only small portions of texts. For example, the blind user who is skipping from link to link with the tab key will read the link text on the braille display or will hear it from the synthesizer (e.g. ".pdf", "more details", etc.). However, the user will not be able to know what is written before and after.

- Information overload due to excessive sequential reading - The static portions of the page (links, frames with banners, etc.) may overload the reading process through a screen reader, since the user has to read every thing almost every time, significantly increasing the navigation time . Thus, it is important to introduce mechanisms to facilitate the identification of precise parts within the page. At the top of the result pages generated by a search engine, for example, the user usually finds several links, advertisements, search fields, buttons, etc., that the user should be able to skip to go directly to the search results listed below. 
Although the Internet is a precious source of information and offers great availability of services, these drawbacks can discourage blind and visually-impaired users from accessing on-line services.

As the previously mentioned screen reader Jaws for Windows is the most frequently used by blind people, in the following we will consider it. Jaws supports quick access to the information in a logical format and also provides further information about the structure and organization of Web pages. Hence, tables, lists, headings, and so forth are all listed vocally. Users can navigate within Web pages via the Virtual Cursor, read web pages using standard screen reader commands, tab through links and easily follow them, display lists of links or headings to quickly find and move to what they need, and easily move to and fill out Web-based forms. For instance, by pressing a letter the focus moves directly to tables, headings, or lists.

To sum up, as already discussed in previous work (Leporini and Paternò, 2004), the main issues in user interface design for vision-impaired users to consider are:

a) Page content serialization. General speaking, the screen reader takes the page source and serializes its content (link, edit field, button, cell, and so on), including frames or blocks $<\operatorname{div}>$, without taking into account specific positions assigned by CSS properties. Basically, the screen reader interprets the code as it was written and arranges the page content in the form of a single column.

b) Navigation by Tab key and special commands. A blind user usually prefers to visit the page link by link (by Tab key) or using special commands in order to move quickly around the pages. Hence, it is important to support keyboard navigation by assigning a scale of importance to the links, applying shortcuts to main elements, using specific tags, such as $<\mathrm{Hn}>$, and so on. Furthermore, many special screen reader commands operate well only if the developer has applied specific tags or attributes, or appropriate criteria have been followed.

c) The information conveyed through visual layouts differs from that afforded by aural perception. Often when developers design a Web page they provide some useful information by means of visual features, such as position, colour, separating blank spaces, formatting features, and so forth. For instance, some secondary information is put on the side so that users can recognize it immediately. It is important to provide the same "message" to a blind user by another means (e.g. using a table, a heading, a hidden label, etc.).

Considering all the above issues, as well as the accessibility and usability difficulties resulting from the screen reader, the identified criteria attempt to structure and organize the content and UI elements in order to allow for better interaction through assistive technology.

\subsection{RELATED WORK}

In addressing accessibility and usability issues, two kinds of approaches can be considered: one takes into account possible methodologies able to make interfaces for all users, which can also interact well with particular devices used by special users; another one is oriented to building "dedicated" interface versions for specific target users. The research community has shown interest in both approaches. Our 
investigation follows the first approach and aims to identify possible solutions in order to make Web user interfaces more accessible and usable for all, including users who are forced to interact by some special devices (e.g. screen reader). Generally speaking, the first type of approach calls for well-defined criteria and guidelines in order to assist designers. Regarding Web interfaces, up to now usability and accessibility guidelines have usually been proposed separately, whereas we intend to consider an integrated approach. Several detailed user interface guidelines were formulated for general user interfaces (for example, (Mayhew, 1992) and (Smith and Mosier, 1986)). Most accessibility issues are currently considered by W3C (World Wide Web Consortium) in the Web Accessibility Initiative (WAI). In the WAI activities, a set of specific guidelines and recommendations has been defined: "Web Content Accessibility Guidelines 1.0" (http://www.w3c.org/wai). Currently, a new version (2.0) of the Web Content Accessibility Guidelines as a Recommendation is in progress (http://www.w3.org/WAI/GL/WCAG20/).

Recently, various international projects have addressed issues related to accessibility or usability of interfaces for users with special needs. Stephanidis' group developed methods and tools for unified user interfaces (Stephanidis et al., 1998). A "Unified Web Browser" was developed as part of the project "AVANTI": it employs adaptability and adaptivity techniques, in order to provide accessibility and high-quality interaction for users with different abilities and needs (e.g., blind users or those with other disabilities). Even if this aspect is related to our research (in fact some checkpoints are similar), the AVANTI browser mainly focused on accessibility and not usability of Web sites accessed by people using screen readers. On the other hand, our intention is to improve Web navigation by applying Web page design criteria, so that people can use a normal browser.

Regarding problems deriving from the visual layout perceived through special devices for the visually-impaired, Donker et al. proposed a method to obtain an aural-based description that has the highest possibility of providing communication effects similar to those of the visual user interface. The approach introduced in (Donker et al., 2002) aims to provide an access not only to page content, but also to other important information provided by the Web page layout. To overcome the layout barrier, the authors concentrated on the possibilities provided through auditory interaction to represent the Web page layout and to support the user navigation. The auditory user interface was tested by involving seven blind users and did not produce excellent results. It revealed that the blind users were not able to process their tasks more effectively and more efficiently with the proposed system compared to their presently used screen reader. Our approach takes into account the difference existing between the visual and the screen reader-mediated perception, but is not aimed at providing specifically aural representation of all the aspects of the visual layout (such as position or dimension). Rather, our method provides the same type of information given through visual layout (position, colour, white spaces, etc.) by using other techniques (e.g. hidden labels, heading levels, etc.).

Brewster's group at Glasgow University has been working in the Multimodal visualization for blind people project (http://www.dcs.gla.ac.uk/ lorna/home.html). The Multivis project has the purpose to build up "visualization" systems allowing people with visual problems to get access to complex information (tables, graphics, structured information etc.) by means of different representation modes, in addition to the classic visualization technique. The goal is to investigate and solve this issue 
by allowing the users to "feel" and "touch" the visualized data. The research leverages previous work about earcons, audio 3D and haptics. Sound was specifically used to aid problems with buttons, scrollbars, menus, progress bars selecting text and files, etc. So, non-speech sounds have been used to improve especially the usability of a graphics package. We think that receiving sounds associated with particular objects, events or task performance states is useful for blind users. Thus, in our work we have also considered non-speech audio in Web site design in order to facilitate the navigation. In particular, we propose to provide different sounds corresponding to diverse events (e.g. differentiating kinds of links through various sounds).

Goble et al. (2000) proposed the use of the model of a real world travel in order to classify aspects in Web sites that are important for usability and accessibility. However, although that study addresses important issues arising from perception of a visual layout in a different manner, they did not provide design guidelines which can be helpful for site and page developers.

Lee et al. (2003) and Hanson et al. (2005) have developed tools which allow enhancing Web browsers capability in order to support a number of interaction techniques, which make user navigation more accessible and usable. This is a useful contribution. However, many users still access through traditional Web browsers and, consequently, there is a need for providing designers and evaluators with guidelines and methods that help them to understand whether the considered sites are accessible and usable. In this direction, Takagi et al. (2004) have proposed a new method, "Blind Usability Visualization". This method aims to enable Web designers to recognize their pages' usability "at a glance", including the timeoriented aspects. It also aids then to assess the usability for blind users. This method represents the time it takes users to reach each part of a page by using background "colours" associated with the time necessary to get from the top to each part of the page.

Regarding the usability of Web sites for users with disabilities, Barnicle (2000) reports on a preliminary testing of the usability of GUI applications through screen readers which revealed many issues. This highlights the need for specific accessibility and usability design criteria, which need to be validated to assess their effectiveness. Even if (Mankoff et al., 2005) stated that working with sighted persons with a good expertise in computing and using a screen reader is one of the most effective methods for testing accessibility problems, we believe that people who usually interact through a screen reader can point out usability problems that cannot be revealed by a person who has no experience with screen readers. (Theofanos and Redish, 2003) report on another study about the usability of Web site navigation through screen readers performed by involving blind users. In particular, this work addresses accessibility supported through the 508 standard. A user testing experiment conducted with 16 blind users showed the lack of support of usability criteria according to the 508 standard guidelines. Based on the empirical evaluation, 32 guidelines were suggested, aimed to improve usability for blind users. Some of these guidelines concern Web site development, while others concern screen reader developers. As a result, an unstructured and unorganised list of guidelines was proposed. Our set of guidelines is different in various respects and here we report on its empirical validation.

In general there is a substantial body of research on Web design for individuals with visual impairments. While this research may not all focus on design 
guidelines, the reader interested in related topics can find other useful sources of information in the proceedings of specialised conferences (such as ASSETS).

\subsection{WEB USABILITY CRITERIA FOR VISION-IMPAIRED USERS}

In order to improve the navigability through screen readers and to make Web sites more accessible and usable for a specific group of disabled users - visionimpaired people - we have identified a set of criteria (Leporini and Paternò, 2004). The identification of such criteria was performed by gathering empirical feedback from vision-impaired users in operating with screen readers, by which potential issues were identified. Subsequently, a systematic method was developed on the basis of the analysis of potential solutions, resulting in a classification of the criteria according to usability aspects. In practise, starting with accessibility guidelines and usability principles, possible combinations of them were identified and empirically tested by some vision-impaired end users (including one of the authors who is blind) (Leporini, 2003) and by developers in applying them to some existing public administration web sites (Leporini and Paternò, 2004).

Our criteria do not represent the only attempt to provide a practical solution to accessibility and usability problems. Indeed, (Theofanos and Redish, 2003) proposed 32 guidelines for accessibility and usability when navigating through a screen reader. Although these guidelines are similar to ours in that they address navigational difficulties for blind people, they are formulated for specific case studies. They are mainly focused on how text parts (e.g. sentences or text included in forms) or specific elements (e.g., links) should be written, whereas our criteria are more general principles for designers by which specific cases can be obtained. Moreover, our criteria aim to consider navigation issues not only through a screen reader, but also by a magnifier program. On the other hand, (Fukuda et al., 2005) have proposed possible metrics to evaluate accessibility in usability terms. Those metrics are classified according to two main aspects: navigability - which evaluates how well structured the Web content is - and listenability - which denotes how appropriate the alternative texts are. If compared to our criteria, the guidelines briefly described in (Fokuda et al., 2005) focus only on the time required to read a page (navigability) and the suitability of the text used for alternative descriptions or the presence of repeated text (listenability). Even if some of our criteria may be similar to the features considered by (Fokuda et al., 2005), our aim is to suggest a general set of criteria that developers should apply when designing Web pages by addressing a wider range of situations (e.g. logical partition of elements, specific sections, and so on).

Each criterion can include one or more checkpoints. Checkpoints are technical solutions that allow the application/evaluation of our criteria and usually correspond to specific implementation constructs that guarantee the satisfaction of the associated criterion. In fact, usually several solutions can be adopted. For example, the page content could be structured by using frames, or blocks $<$ div $>$ customisable by css properties. Alternatively, the page content could be visualised by embedding it in layout or data tables. An example application of such criteria is described in section (3.4).

In a first phase (Leporini and Paternò, 2004) the identified 19 criteria were grouped into three sub-sets: (1) effectiveness, (2) efficiency, (3) satisfaction. Then, we have reorganised them into a more accurate logical structure, obtaining 15 criteria grouped into four categories according to structure and arrangement, content appropriateness, multimodal output, and consistency issues. Many criteria 
visually affect the Web interface (e.g. coloured areas or element magnifications), whereas others are exploited by the screen reader (e.g. hidden labels or names of frames). The criteria are reported in the following and labelled with the letter " $\mathrm{S}$ " if they refer specifically to screen reader navigation, with the letter "M" if they are linked only to magnifying features and with the letter " $G$ " if they refer both to screen readers and magnifiers (general use).

\subsubsection{Structure and arrangement}

This set of criteria is aimed at organizing and structuring the user interface elements and the page content in order to make the navigation easier. The main difficulties that should be solved through these criteria are the user's orientation and the ability to obtain a page overview. The criteria of this set are:

- Logical partition of interface elements (1.1) (G) This criterion aims to group information, links, fields and so on in logical categories in order to help users reading a page through screen readers to locate the essential parts. Moreover, some screen readers allow skipping from section to section. This can be obtained by using markers, frames or headings to group texts, links, forms, and so on, according to a logical division. In addition, in order to more easily reach some specific content in the page (or in the site) developers can insert local navigation links referring to bookmarks within the scope of the page (e.g., 'skip to content', 'go to top', 'go to navigation bar', etc.). We also suggest adding a local index, such as local page links, in pages containing information of different kinds (paragraphs, news, etc.) in order to navigate more efficiently among the page sections.

o Number of links and frames (1.2)

It is important that a page does not contain too many links or frames, as this makes it difficult for the user to skim through them. Too many links take a long time for readers to get through; too few links may imply too many levels in the Web pages' hierarchy structure. The suitable number of links depends on the page role in the corresponding site.

- Specific sections (1.3) $(G)$

Web pages should allow users to quickly identify specific site sections. Pages should have clearly marked the navigation bar, menu or submenu, main content, last updates and so on. The navigation bar (i.e., the links appearing on each page and enabling users to reach the main parts of the site) represents a source of delay and inefficiency for screen reader users. Since such links appear on each page (and often even twice), the users who are forced to read the content in an almost sequential way are always compelled to access them before identifying the page contents. Therefore, well-structuring and highlighting the navigation bar at the top and/or the bottom of the page, if any, can be useful to make it more understandable to vision-impaired users and can increase their navigation efficiency.

In sites with frequent information updates and/or new resources to download, we can help the user find the new elements more rapidly by providing a specific section listing the new elements by date, thereby sparing the user the trouble of useless navigation. Furthermore, a specific page listing all short keys associated to the most important links of the Web site should be considered. With respect to the criterion 1.1, here we 
refer to the fact that certain precise sections should be available on the Web site (e.g. last updates section).
o Importance levels
of
elements

In order to facilitate navigation, especially when using the keyboard, it is possible to assign different importance values to interaction elements such as links, buttons, and fields. Thus, when users move through the 'Tab' key (element by element), they visit at first the most important, and later the less important, regardless of their location on the page.

\section{o Proper \\ form \\ layout

In forms with several groups of input data, we must properly lay out group titles and fields to achieve a major clearness. In fact, the way the elements are formatted can cause confusion with the screen reader. For example, in some cases the voice synthesizer or Braille display could read before the 'checkbox', 'combobox' or 'field' item, and after its value, or vice versa. Thus, a correct application of layout elements (e.g., simply by using the label tag in the proper place) is recommended.

o Assignment of shortcuts (1.6)

It is advisable to assign shortcuts to access the most important buttons, links and fields, so that the user is able to reach them quickly through a simple key combination. This feature may be useful especially when users visit the Web site frequently, and learn the key combinations by heart.

\subsubsection{Content appropriateness}

When perceiving content through a screen reader, especially by using a vocal output, making the content clear and auto-explicative is very important. In fact, the global overview is loss, and usually a blind user moves through the Tab key or screen reader special commands in order to navigate fast. This means that the user reads the content quickly and jumps from link to link, from table to table, and so on. For this reason, it is important to provide an appropriate content for textual and graphical links, for table summary, for pictures and images, paying particular attention to those with a functional purpose rather than a decorative scope. Criteria included in this group are:

o Proper link content (2.1) (S)

The link labels are important for special users who use screen readers and keyboard commands. Thus, the links should be clear and context independent, and not having general texts such as 'more details', 'download', '.pdf'. We must warn the designer that such texts can lower the site's usability for blind users, because they are ambiguous, or not enough informative. In addition, the link content should not start with the same letter or symbol - such as links starting with the words "link to...", or character "[", etc. - because it reduces the effectiveness of the navigation by the initial letter when the list of available links is opened using a specific screen reader command (Insert+F7).

o Proper name for frames, tables and images (2.2) (S) It is important to check that all frames, tables and images have names and descriptions that are appropriate and meaningful; e.g., frames with names such as "top" are not very helpful to the user. On the other hand, names such as "index" can make it easier for users to reach their goals. Similar considerations apply to text summaries of tables and to alternative description for images and graphics. Descriptions such as "This table is 
used for a layout purpose..." are not particularly useful since its reading aloud by the voice synthesizer risks to be tedious.

\subsubsection{Multimodal output}

The goal of the criteria belonging to this set is to provide blind or low-vision users with several kinds of feedback. Using different colours, changing colours or dimension for focusing the current content, adding different sounds can help the visually-impaired user. In fact, particular visual or sound cues can represent good feedbacks for user's orientation.

o Messages and dynamic data management (3.1) (S) A significant difficulty often encountered with screen readers is represented by poorly designed system feedback. This may be confirmation or error messages about operation commands or information requests, which are not presented in a manner that can be rapidly interpreted by screen readers (e.g., messages in the middle of the page or amongst a lot of other information), or they are often so "short" that they are easy to miss. This principle suggests paying attention to this aspect by applying certain features to facilitate identification of new messages or data.

o $\quad$ Loading suitable style sheets (3.2) $(G)$

Browsers can load specific sheets for different output devices. This style sheet feature allows specifying how a document should be presented on different media, such as screen, paper, speech synthesizer, Braille device, etc.

o Addition of short sounds (3.3) (S)

Associating a short sound with different elements can provide useful feedback. E.g., providing each page with a short sound indicating when the loading of the page is completed eliminates the need for the user to repeatedly check the status bar.

- Colour of text and background (3.4) (M)

For low-vision people it is advisable to avoid colour combinations that provide poor contrast. Furthermore, changing colours in correspondence to some events or particular areas can be a way to get attention.

o Magnification at mouse hovering (3.5) (M)

The use of this feature can help people with a good visual residue to better focus on the object beneath the cursor. The idea is to enlarge interactive elements such as navigation bar and buttons, but not all the text because it would be confusing.

\subsubsection{Consistency}

Keeping the content and layout consistency across Web pages is in general important for the user. This is particularly significant for visually-impaired users because they rely on consistent features.

- Layout and terminological consistency (4.1) (G)

Consistency is a usability feature that allows users to better understand the context and the available functions. It is important that all the pages of the whole Web site adopt the same labels for buttons performing the same tasks (e.g., OK/Yes, quit/exit), and that all pages have the same layout (e.g., dimension, form and colour). 
o Page information (4.2) (S)

Defining the beginning and end of the page by adding useful information can make the navigation more pleasant. For instance, the title of the page, which is read as first line, could contain not only the Web page title, but also additional indication such as the current path in the Web site. The last line of the page could contain information that allows users to understand that they have reached the last line.

\section{THE USER TESTS}

\subsection{OVERVIEW}

In order to evaluate the impact of our identified criteria on Web navigation by visually-impaired users, we conducted two separate tests. Two Web sites were developed and two groups of end users (visually-impaired) were recruited in order to evaluate them. A remote user testing had been conducted in order to collect all data. In each test users were asked to perform seven tasks indicated by a "Wizardbased method" (See section3.5).

The same protocol - better described later - was used for both tests. More detail about how the experiments were performed is provided in the following sections.

\subsection{METHOD}

\subsubsection{Participants}

For each Web site a specific test was carried out: $\mathrm{Test}^{\mathrm{A}}$, with a first group of twenty users (10 blind and 10 low-vision), and Test ${ }^{\mathrm{B}}$, with a second one composed of twenty users too (14 blind and 6 low-vision). We use Group ${ }^{A}$ and Group ${ }^{B}$ to refer to the user group involved respectively in the Test ${ }^{\mathrm{A}}$ and in Test ${ }^{\mathrm{B}}$. All the users needed navigate supported by a screen reader. In particular, the low -vision users included in our experiment, although being able of use some visual features to orientate within the page (e.g. colour changing), relied substantially on a screen reader for operating due to their serious visual deficit.

The user age varied from 25 to 68 years. All the participants had been using Windows 98/ME/XP and Jaws (as screen reading application) for at least one year at the moment of the testing and most of them learned to use the computer through screen reader by attending targeted courses given by the association for the blind. According to the questionnaires, $70 \%$ of the users used Windows application on a regular basis and only $20 \%$ could be defined navigation experts. About half of the people used to navigate Web sites daily and the other half at least two days for week.

\subsubsection{Experimental protocol}

Not all the users knew the more advanced screen reader commands and functionalities (e.g. commands for listing available links, all heading levels in the page, skipping table by table or heading by heading, and so on). For this reason, the experimental protocol was divided into two phases.

Preliminary phase: participants were provided with a list summarising the most important Jaws commands and were allowed to explore both Web site versions (with and without our criteria) for a week before the testing. Users were asked to navigate the two Web sites and to fill in and send forms at least three times. 
Testing phase: our testing procedure was based on remote evaluation, which is the evaluation performed when evaluators and users are separated in time and/or space. Remote evaluation allows designers to analyse the performance of users who interact with applications in their familiar environment. An unfavourable aspect found in our remote evaluation method regards the setting of the client-side environment required for the testing. In fact, the browser logging tool requires that the java VM be installed. We noticed that some users had difficulties in downloading and installing the java plug-in.

Two remote evaluation techniques were included in the procedure: client-side automatic logging and remote questionnaire.

- The task-based logged session was subdivided into two parts, one conducted on the "control site" ("session $(c)$ ") and one on the "implemented site" ("session $(i)$ "). The two tests were performed online, so that users were able to connect to them from their own computers. Users' interactions were automatically collected during the test. Half of the users were asked to start from "session $(c)$ " and the other half from "session $(i)$ ".

- The remote questionnaire aimed to evaluate qualitative aspects of Web site usability, such as user satisfaction or enjoyment. In order to not influence the users, we decided to submit the questionnaire just after the two tests had been carried out: when users finished the two tests, they received a document containing the 18 questions by e-mail.

The two different techniques allowed us to collect two kinds of data: Objective Time spent by users performing assigned tasks; Subjective- Users' preferences, opinions, and suggestions.

The experimental protocol was designed to avoid two kinds of bias:

- bias of ability: discrepancies in navigation abilities within users, associated to a different degree of individual training, could affect the result of the test: the preliminary phase allowed the participants to start the testing procedure with similar basic skills especially regarding knowledge of Jaws commands;

- bias of familiarity: extra navigation familiarity gained by users during the first test could influence the results of the subsequent test (e.g., users could become quicker in performing tasks during the second test). Preliminary navigation on both Web site versions reduced the surplus of experience possibly gained during the first test. Furthermore, the reversed order of test performance in half of the participants $\left(\operatorname{session}_{(\mathrm{c})}\right.$-session $_{(i)} / \operatorname{session}_{(i)^{-}}$ $\left.\operatorname{session}_{(c)}\right)$ contributed to smooth the effect of possible residual bias of familiarity. In any case, although the tasks assigned for the two tests belong to the same typology (e.g. searching for an information, downloading a file or document, and so on), they are different: e.g., the task is "searching for" but the argument differs from session $(\mathrm{c})$ to $\operatorname{session}_{(\mathrm{i})}$.

\subsubsection{Logging Tool}

An automatic logging tool was used to record the time spent by users carrying out the assigned tasks. Our tool is an adaptation of the logging tool which was developed for the WebRemUsine tool (Paganelli and Paternò, 2003). The main interaction activities performed by each user during the testing procedure were captured and logged. 
The tool included Javascript functions, java applet, and java servlet. The tool component in Javascript was able to detect all user interactions with the browser (client side). Then, all the events detected were passed to a Java applet (client side). The applet allowed gathering all the interactions provided by the Javascript component. At the end of the testing procedure, the applet sent all data to a servlet that stored the logs file (server side).

Such log files contained a wide variety of user actions (such as mouse clicks, text typing, link selections...) as well as browsing behaviour, such as page loading start and end. In particular, the tool logged the time when a specific interaction was performed. Time was expressed in milliseconds and then converted into seconds. During the testing procedure, users had to click on the "Next task" link when they switched to the following task.

\subsubsection{The Questionnaire}

After the end of both tests, participants were asked to fill in a questionnaire composed of 18 questions. Such questionnaires allowed collecting information about the operating system and the assistive technologies used by the participants, and to obtain other qualitative data not obtainable by the logging tool. Subjective information was also considered. For example, users could express opinions and ideas about the usefulness of sounds, shortcuts, etc. In addition, a section was specifically designed for low-vision users, in order to register opinions on colour contrast and link magnification. Indications about the level of difficulty of each task were also considered. Lastly, suggestions and comments were elicited.

\subsection{THE TEST WEB SITES}

For our tests, we considered two Web sites. The first one containing specific information about the "The Tuscan Association for the Blind" (Unione Italiana Ciechi - Regione Toscana) was chosen with the intent of providing blind people with a comfortable situation with familiar information, thus reducing navigation difficulties. The second Web site contained information about a publishing house. We decided to use two Web sites in order to applying the identified criteria to different sites with different visual layouts. Since each criterion can be applied by various checkpoints, we used different Web sites in order to consider diverse cases. More precisely, in the first site a horizontal navigation bar and a vertical side-menu were used while in the second one two vertical side-menus were used for the layout (i.e. by using a layout with three columns). Thus, the two Web sites allow us to better evaluate the various modalities of criteria application through the selection of different checkpoints.

In both cases, we created two versions of the same Web site: one version was implemented according to the 15 criteria and for this reason it will be referred as the "implemented site", while the other version, without criteria, was used as control in our testing protocol ("control site"). In both cases, both versions were technically accessible for vision-impaired users. This means that all the technical accessibility barriers were removed from the pages (i.e. all tags and attributes required by accessibility guidelines were applied). Thus, assistive technologies were able to work with the Web pages. For instance, all images or graphical links had the needed attribute alt with a certain description (e.g. alt="link1", "figure", etc.). Nevertheless the user could still encounter difficulties in orientating within the page content. 
The time required for performing the same kind of tasks in both cases was recorded. Group ${ }^{\mathrm{A}}$ users performed seven tasks on the control Web site ( $\operatorname{Site}^{\mathrm{A}}{ }_{(\mathrm{c})}$ ) and on the implemented version ( $\left.\operatorname{Site}_{(i)}^{A}\right)$; Group ${ }^{B}$ users carried out other seven tasks on the "control" and the "implemented" Web site $\left(\operatorname{Site}^{\mathrm{B}}{ }_{(\mathrm{c})}\right.$ and $\left.\operatorname{Site}_{(\mathrm{i})}^{\mathrm{B}}\right)$.

\subsubsection{The Web site for Test ${ }^{A}$}

The Web site had three main sections, i.e. "News", "Documents\&Download", and "Organization". Each section was reachable from each page and was composed of a variable number of subsections. The general layout of the page included (an example is shown in Figure 1):

- a horizontal navigation bar at the top;

- a vertical submenu or local index at the left;

- $\quad$ the current page content at the right (it is the largest area);

- navigation links ("Go back", "Go to navigation bar", etc.) at the bottom.

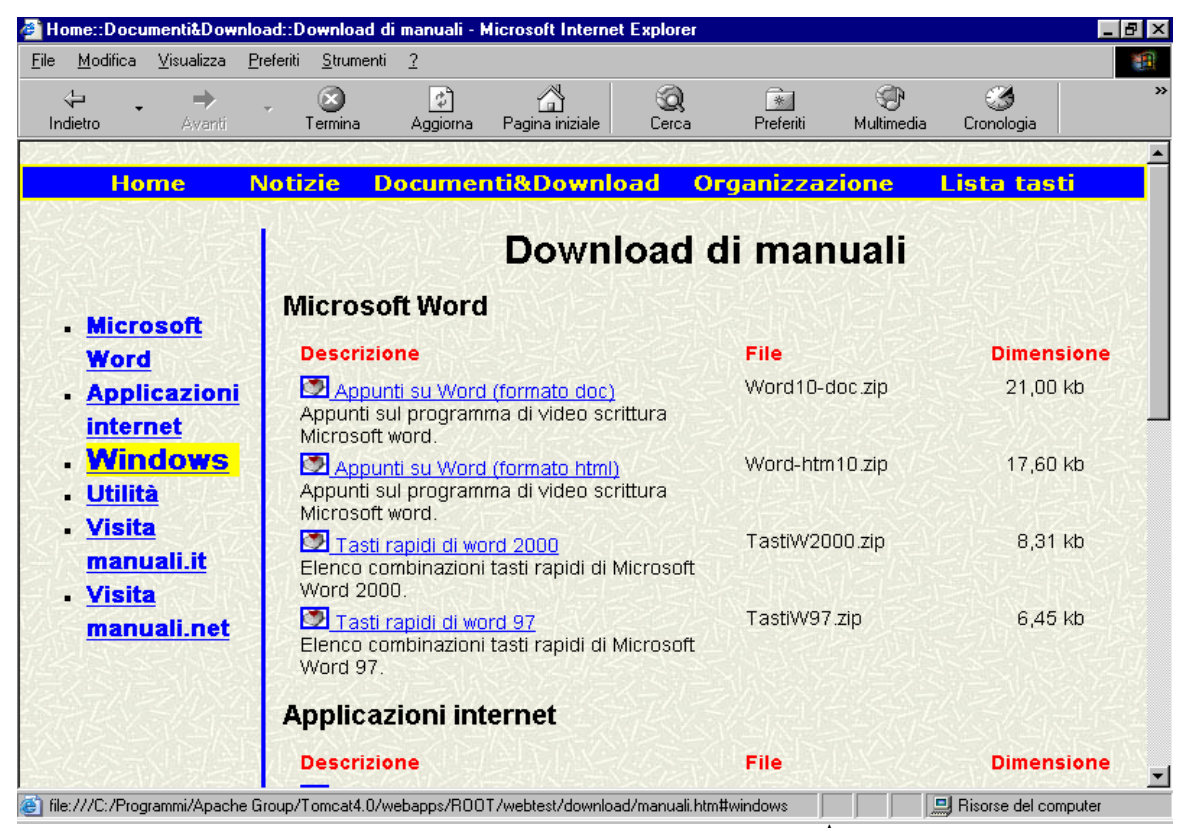

Figure 1 - A Web page used for the Test ${ }^{\mathrm{A}}$.

The Web site is aimed at collecting several information and services that can be useful especially for blind and low-vision people as well as for all those who like to get information on vision issues. Thus, the Web site provides information such as a periodical bulletin with several news; download sections containing various documents and local and external files (e.g. utilities, screen readers demo versions, etc.); some catalogues regarding works available in braille format; some information related to the structure and organization of the association and so on. All these data are organized in several sections, which can be accessed through the navigation bar. 


\subsubsection{The Web site for Test ${ }^{B}$}

When the second test was performed, a Web site related to a publishing house was considered: "Equilibri editore". This Web site provides general information on the books printed, on their authors, and so on. Books are listed by titles, by authors and are arranged also by category. Biographical information on the authors who publish for the publishing house as well as several interviews are available. A News section about forthcoming books is published on the Web site as well.

Links pointing to all the specific sections (e.g. titles, authors, categories, etc.) are arranged in two side menus: on the left links to book search sections (by title | authors | category) are available; on the top-right of the page, more general links (such as about us) are listed in the vertical menu.

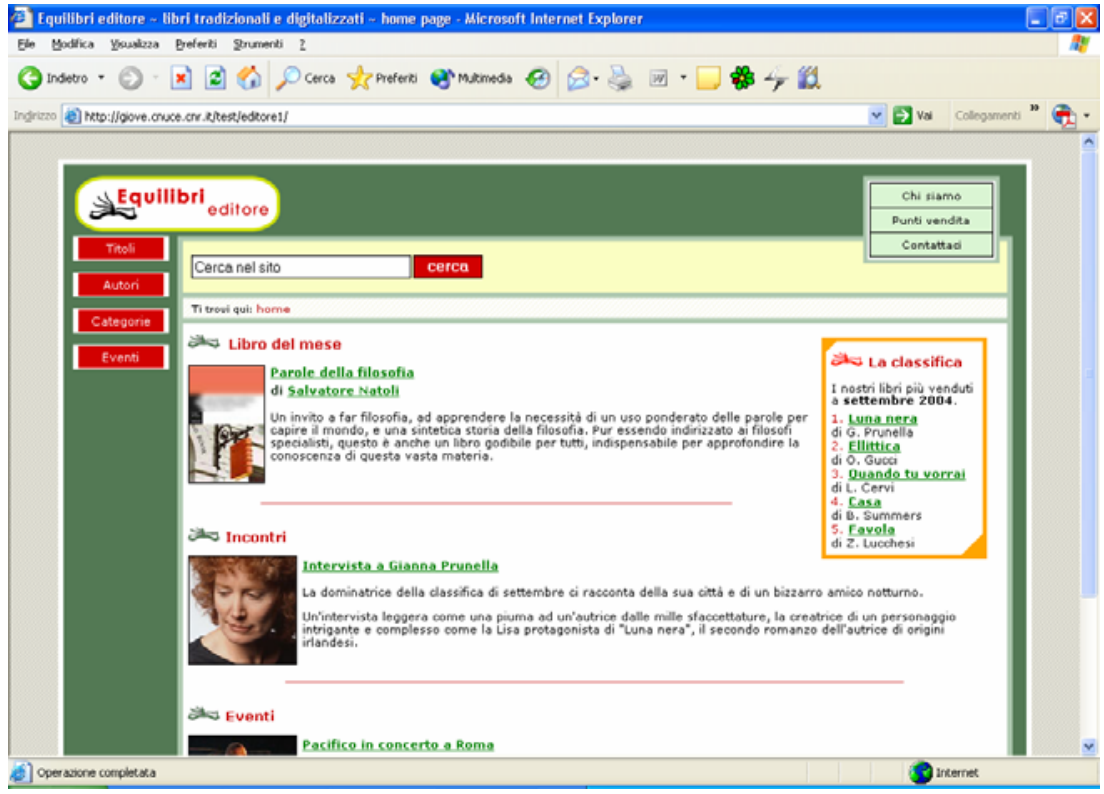

Figure 2 - The home page of the Web site used in the Test ${ }^{\mathrm{B}}$.

Figure 2 shows the home page of the "Equilibri" publishing house, the layout of the page contains:

- A left-side vertical menu, i.e. the links pointing to the sections related to the books (i.e. titles, authors, etc.);

- A right-side vertical menu, composed of general links (about us, Where we are, etc);

- A main central page section containing the information related to the current page.

Div blocks and CSS properties were used for implementing the visual layout; in this way, the left and right side-menu are interpreted sequentially by the screen reader. Figure 3 shows an excerpt of the implementation and how it is rendered by the screen reader.

\begin{tabular}{l|lll}
$\begin{array}{l}\text { Link skip to content } \\
\text { Graphic Equilibri }\end{array}$ & $\begin{array}{l}<\mathrm{p} \quad \text { class }=\text { "invisibile" }><\mathrm{a} \\
\text { content }</ \mathrm{a}></ \mathrm{p}>\ldots\end{array}$ & href="\#dove" $>$ Skip & to \\
\hline
\end{tabular}




\begin{tabular}{|c|c|}
\hline $\begin{array}{l}\text { editore - home page } \\
\text { Heading level } \\
\text { Navigation bar: } \\
\text { List of } 4 \text { items } \\
\text { - Link Titles alt }+\mathrm{t} \\
\text { - Link Authors alt }+\mathrm{a} \\
\text { - Link category alt }+\mathrm{k} \\
\text { - Link Events alt }+\mathrm{e} \\
\text { list end } \\
\text { List of } 3 \text { items } \\
\text { - Link About us } \\
\text { - Link Sale points } \\
\text { - Contact us } \\
\text { list end }\end{array}$ & $\begin{array}{l}<\text { img src="img/logo01.gif" alt="Equilibri editore - } \\
\text { home page"/> } \\
<\text { h1 class="invisibile" }>\text { Navigation bar: }</ \text { h1 }><\text { div } \\
\text { id="navigazione" }> \\
\quad<\text { ul }> \\
<\text { li }><\text { ahref="titoli.html"accesskey="t" }>\text { Titles }</ \text { a }></ \text { li }> \\
</ \text { ul }> \\
\ldots \ldots \\
</ \text { div }><\text { !-- left side-menu --> } \\
<\text { div id="navigazione2" }> \\
<\text { ul }> \\
<\text { li }><\text { a href="chisiamo.html" }>\text { About us }</ \text { a }></ \text { li }> \\
\quad \text { ul }> \\
\ldots \ldots \\
</ \text { div }><\text { !-- end of right side menu }-->\end{array}$ \\
\hline
\end{tabular}

Figure 3 - Side menus: screen reader interpretation (left), and excerpt of the XHTML code (right).

\subsection{APPLIED CRITERIA}

As already mentioned, two versions were developed of each Web site: one was developed without applying the usability criteria, whereas the other one was built by following the criteria to be tested. Application of the criteria to one of the two versions of the test Web sites involved the aspects summarised below according to the four groups.

\subsubsection{Structure and arrangement}

- Logical partition. Heading levels were often used to structure the information in a logical way, but in some cases hidden labels or tables with summary values were also applied. For instance, navigation bar and submenu were marked with appropriate labels (e.g. "navigation bar:" and "submenu:"). Then, in pages containing various file information and links related to downloadable manuals or programs, each data group was placed in a specific table with group names as summary attributes.

- Number of links and frames. The Web sites were built with a limited number of links per page and without frames.

- Location of the navigation bar. In the first prototype, Site ${ }_{(i)}{ }$, both navigation bar (at the top of the page) and a submenu (at the left size of the page) were included together with hidden labels "navigation bar" and "submenu" to mark bar and menu beginning. In the Site ${ }^{\mathrm{B}}(\mathrm{i})$ the "Navigation bar" hidden label was added before the side menus. In addition, the vertical menus belonging to both Web sites were built by including item links in a bullet list: in this way users were able to identify its structure more quickly (e.g., by pressing the "l" key when interacting through the screen reader).

- Specific sections. In the first Web site, "Last updates" and "Key list" sections were added. The "last update" section was reachable from the home page, whereas the "Key list" page could be found in the navigation bar of each 
page. Differently, in the second one, no specific page containing the shortcut list was added to the site.

- Content Indexing. In the two sites, two types of content page indexes were applied: (1) a list of local links was added when a page contained several blocks of information; (2) a drop down menu was added when a page contained many block of information identifiable by characters from "a" to "z" (e.g., a list of song titles). A similar solution was applied to the page created for collecting a set of documents organised by date. In this case, the index was built by assigning a specific menu item to each group of documents belonging to the same year. In short, the first type of index was used when only few links were necessary to build the local index, while the second type was applied when a high number of links was needed.

- Different visiting order of elements (Importance levels of page elements and Keyboard shortcuts). In each page, except for the home page, the lowest visit order value was assigned to navigation bar links (i.e. the highest tabindex value), whereas greater priority was assigned to the submenu and other links. In this way users could first visit links associated to submenu/more recent information and afterwards go to navigation bar links. Navigation bar/link shortcuts were also associated to navigation links. For instance, in both Sites $^{\mathrm{A}, \mathrm{B}}{ }_{(\mathrm{i})}$, Alt $+\mathrm{h}$ was used to go to the home page.

- Navigation links. Links such as "skip to content", "go top of page", "go to navigation bar", "go to submenu", and "go back" were added to each page to facilitate the navigation. Furthermore, the two links "go to navigation bar" and "go to submenu" were made "invisible", since they are useful only for navigation through screen reader or keyboard.

- Proper form layout. A specific page, built by using appropriate tags, contained a form to fill and send for obtaining information, make suggestions, etc. Besides, CSS properties (and not tables) were used to render the set of pairs $<$ label, edit field $>$.

\subsubsection{Content appropriateness}

- Proper link content. For textual links "title" attributes were used when link labels were not appropriate by themselves; in the other cases, graphical and textual links were used together. For instance, a graphical link with an appropriate icon was combined with an appropriate text to obtain a download link: the word "download" was assigned to "alt" attribute to graphical link, and the name of the file to be downloaded was used as label of the link. In this way, the visual link is represented by a symbolic icon close to the name of the downloadable program (e.g., Download Acrobat Reader 5.1). In particular, in the left and right menu links available in the Site B, graphical links were used. In the control version of the Site ${ }^{\mathrm{B}}$, an alternative text such as "Link1", "Link2", and so on, were assigned to graphical links "Titles", "Authors", etc.. Consequently those contents were not significant, but the page was still technically accessible (i.e. the screen reader was able to capture the alternative descriptions). Then, in the implemented version a more significant text was assigned (i.e. Titles, Authors, etc.).

- Proper name for frames, tables and images. In the Web sites, style sheet properties were mainly used for organizing the content in the page. In the $\operatorname{Site}^{\mathrm{A}}{ }_{(\mathrm{i})}$ tables were used only to render data about download file information (e.g. programs, manuals, documents, etc.). A table was used to group set of files 
belonging to the same category, in order to obtain a table for each category. In this way, the "summary" content could be represented by the name of the category itself. By pressing the " $t$ " key when interacting through the screen reader, it is easy to skip table by table, which means category by category.

\subsubsection{Multimodal output}

- Messages and dynamic data management. A new page was used to provide information about the status of a form submitted.

- Addition of sounds. Only in the first Web site, Site ${ }_{(i)}^{\mathrm{A}}$, different sounds were associated with the selection of different types of links (i.e. local, internal to the site, external to the site).

- Visual features (Colour of text and background, and Magnifying at passing by mouse). We used different colours for distinguishing navigation bar, submenus, and links currently pointed. In the first site, the navigation bar was arranged horizontally at the top of the page in a blue area; the submenu links were placed vertically at the left of the page and separated by the page content by a vertical black line. When the mouse was over the links, link size and colour changed. Regarding the second Web site, vertical navigation menus are placed in an area having a background colour (green) which differs from the prevailing section colour (white).

\subsubsection{Consistency}

- Layout and Terminological Consistency. All links, buttons and pages had the same features within all pages. In addition, all pages had the same template: the Site $^{\mathrm{A}}$ (i) has the navigation bar at the top, the left side submenu (when necessary), the standard size and colour buttons, and so on; the $\operatorname{Site}^{\mathrm{B}}{ }_{(\mathrm{i})}$ has a left-side and a right-side menu, a search field and a central main page section are applied. Furthermore, the terminology was maintained within the pages of both Web sites, such as "Homepage".

- Additional information. Information on the pathway for reaching each page was indicated. In the $\mathrm{Site}^{\mathrm{A}}{ }_{(\mathrm{i})}$, the page path was included in the $<$ title $>$ tag, e.g., for the program downloading page the title was: "Home :: Documents\&Download :: Program downloading". Instead, in the second site, the path was reported within the page, such as "you are in: Home : Title". However, a short indication about the current page was also inserted in the $<$ title $>$ tag (e.g., Equilibri editore $\sim$ Titoli). Furthermore, each page had the "last update information" on the last line. Figure 4 shows an example of Web site page used for the Test $^{\mathrm{A}}$ : the navigation bar, submenu, and current link magnification are also displayed. It also contains a list of song titles in alphabetical order. The titles are grouped by letter, each of which is associated with a heading level $<\mathrm{H} 3>$. 


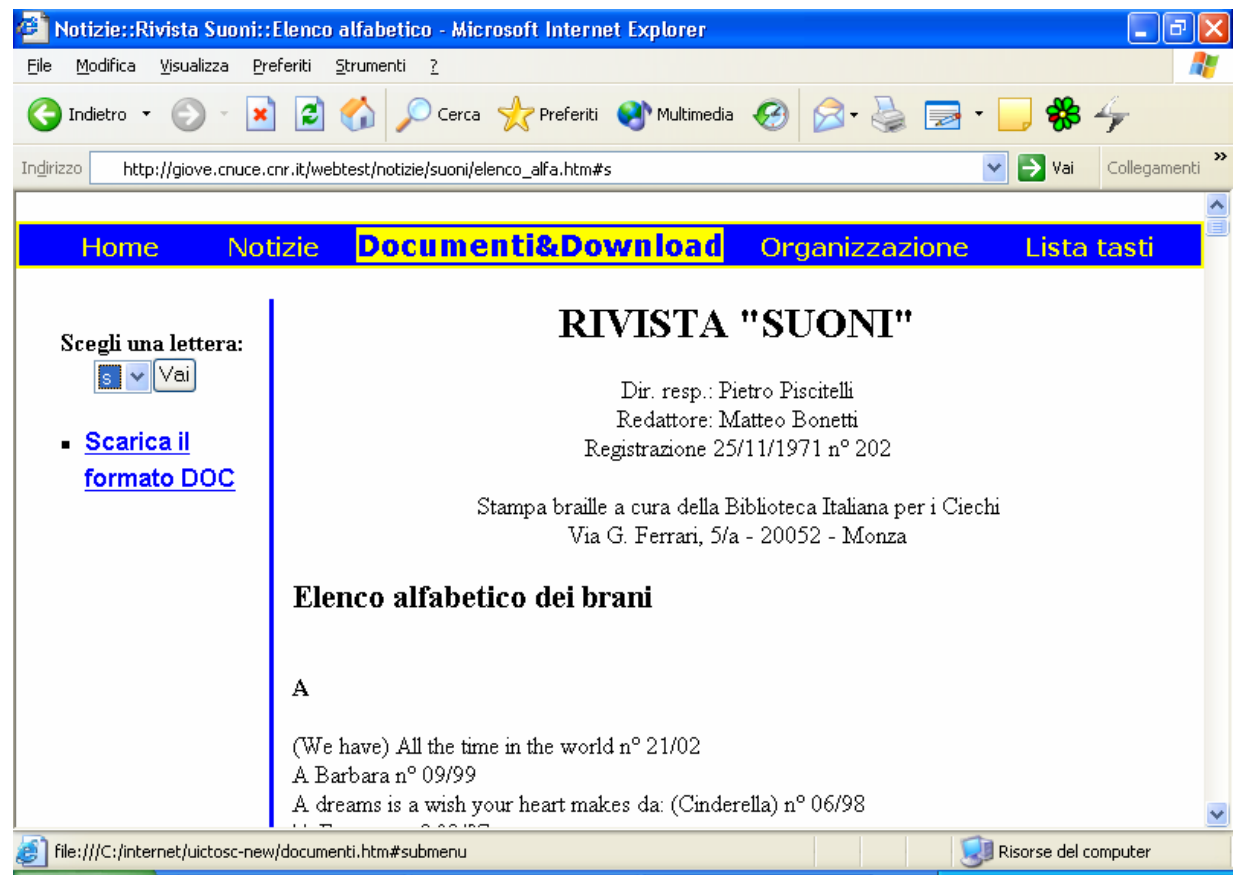

Figure 4 - Page showing a list of song titles divided by headings and with a side list to facilitate navigation.

Figure 5 reports a portion of the heading list generated by a screen reader command applied to the page shown in Figure 4. This means that blind users can go directly to the titles beginning with any given letter by accessing the headings through a specific screen reader command. Then, they can select the characters of interest (in this case "s"). For low-vision users, a drop-down menu has been inserted (on the left side), which allows character selection by mouse as well. This solution is preferable to creating a link for each character (as is often done), because the twenty-six links generated would make navigation more difficult for those interacting through a screen reader and Tab control. 


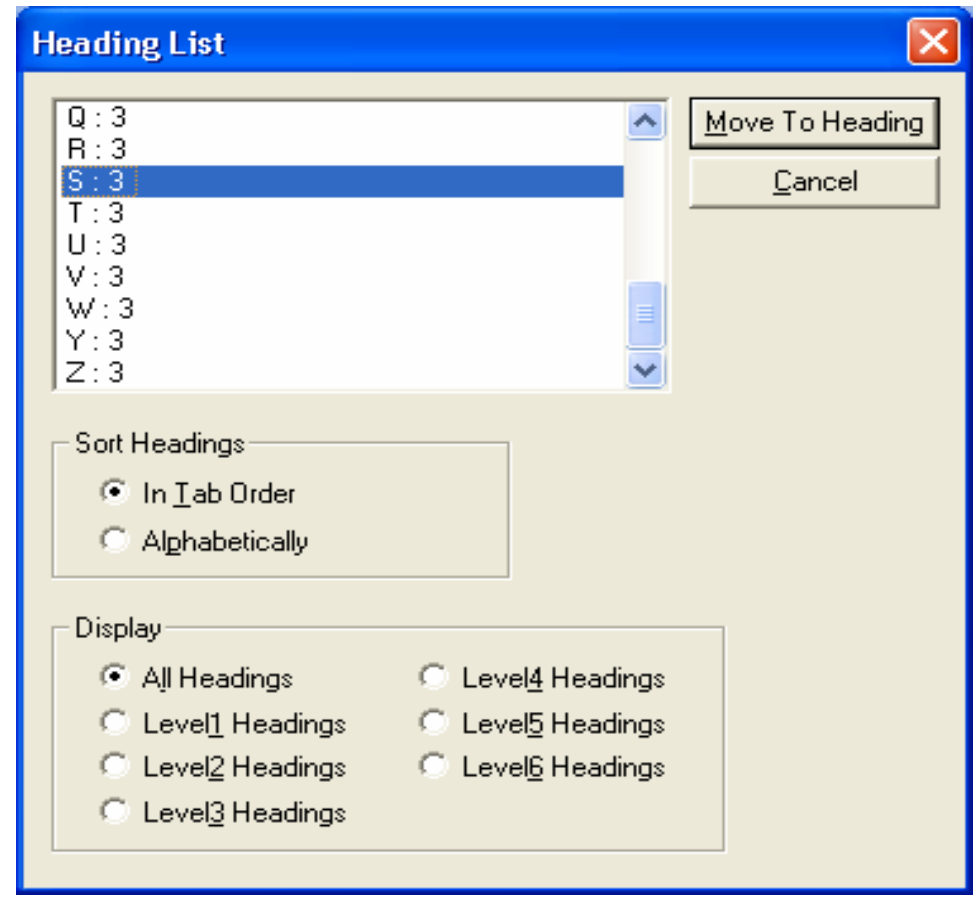

Figure 5- List of heading levels generated by a screen reader command allowing users to jump to the lettered group.

Lastly, Figure 6 shows an excerpt of text generated by the screen reader that highlights some criteria that have been applied. The first row read by the screen reader corresponds to the $<$ title $>$ attribute, which contains the path to the page (e.g. News::Sounds Magazine::Alphabetical List), as well as the title of the page. Such a solution enables users to navigate more easily because the information is immediately read by the screen reader (or in any event, is rapidly found by the screen reader because it corresponds to the first row). The blind user can then check that the screen reader has identified the hidden labels ("navigation bar" and "submenu") used to identify the various components of the page.

\begin{tabular}{|l|c|}
\hline News::Sounds Magazine::Alphabetical List & Equilibri editore $\sim$ Titles \\
Navigation bar: & Link Skip to content alt +0 \\
Link skip to content alt +0 & Link Equilibri - home page \\
Link Home alt $+\mathrm{h}$ & alt $+\mathrm{h}$ \\
Link News alt $+\mathrm{n}$ & Navigation bar: \\
& List of 4 items \\
Submenu: & $\bullet$ Link Titles Alt $+\mathrm{t}$ \\
Choose a letter: & $\bullet$ Link Authors alt $+\mathrm{a}$ \\
Combobox $\mathrm{S}$ & $\ldots$ \\
Button Go & \\
& Search in the site: \\
\hline
\end{tabular}




\begin{tabular}{|c|c|}
\hline $\begin{array}{l}\ldots \\
\text { Alphabetical list of musics } \\
\text { Heading level } 2 \mathrm{~A} \\
\text { (We have) All the time in the world } \mathrm{n}^{\circ} 21 / 02 \\
\text { A Barbara } \mathrm{n}^{\circ} 09 / 99 \\
\text { A dreams is a wish your heart makes da: } \\
\text { (Cinderella) } \mathrm{n}^{\circ} 06 / 98 \\
\ldots \\
\text { Heading level } 3 \mathrm{~S}\end{array}$ & $\begin{array}{l}\text { Edit alt+c } \\
\text { Search button } \\
\text { You are here: } \\
\text { Link Home }> \\
\text { Titles } \\
\text { Heading level } 1 \text { Index of } \\
\text { book titles: } \\
\text { Choose a letter: } \\
\text { Combobox A } \\
\text { Go Button } \\
\text { List of 26 items } \\
\text { • Heading level } 3 \text { A } \\
\text { List of } 1 \text { items nesting } \\
\text { level } 1 \\
\text { - Link Arte povera } \\
\text { di E. Fardelli } \\
\text { list end nesting level } 1 \\
\text { • Heading level } 3 \text { S } \\
\text { List of } 3 \text { items nesting } \\
\text { level } 1 \\
\text { • Link Casa } \\
\text { di B. Summers } \\
\text { • Link Cavallo pazzo } \\
\text { di C. Erri } \\
\text { • Link Cuor di leone } \\
\text { di N. Savoldi } \\
\text { list end nesting level } 1 \\
\ldots\end{array}$ \\
\hline
\end{tabular}

Figure 6 - Part of one page read by the screen reader (translated in English): on the left related to Site $^{\mathrm{A}}$ and on the right to Site $^{\mathrm{B}}$. The parts in italics are read by the screen reader but do not appear on the page shown by the browser.

As shown by our examples, when applying a criterion various technical solutions can be used. For instance, for partitioning the interface elements we mainly used a set of heading levels; in some cases tables or hidden labels were used for grouping the information in a different way. In any event, designers can apply the solutions they prefer, provided that they comply with the general principles expressed by the criterion.

\subsection{TASKS}

A Wizard procedure was used during the test. It consisted in providing users with a standardized indication of the next task to perform without constraining their behaviour. A Wizard is a special form of user help that provides users with step-bystep instructions to complete clearly structured tasks (Marion, 2000). This is done through dialogue with the user. In our experiment, the instructions were provided 
automatically through specific dialogue boxes, which helped users to carry out the test by indicating the successive task.

Participants were asked to carry out seven tasks per test. The tasks included common navigation operations, such as page opening, content reading, and information search. The participants were also required to download files, fill in a form, and so forth. Session $(c)$ and Session $(i)$ included the same types of tasks, which differed only in some minor aspects (e.g. the file to download, the information to find, etc.).

The following list of tasks were assigned to the users, in both $\operatorname{Session}_{(c)}$ and Session $_{(i)}$ in the test A:

1. Visit the bulletin page of Tuscany.

This was a reading content page where the information was organised by using 'heading levels'. The user was asked to look for a specific piece of information.

2. Visit the Web page "Departments and Commissions".

This page was a content reading page where 'heading levels' were used to logically group the different pieces of information. A page index was composed of a small set of local links. The user was asked to find the name of the coordinator of the commission of a specific association.

3. Visit the alphabetically ordered list of "Sound Magazine". This page was a content reading, where both 'heading levels' and an index combo-box were used to organize page information. The user had to search for a specific song title.

4. Downloading a document

This page was a content page containing a specific link for downloading an application form. The user had to search and download a specific application form.

5. Download a zip file.

This was a 'downloads page' where groups of files were placed in several tables: each table summary contains the name of a specific group. The users had to download a specific file.

6. Open an external URL.

This was an 'index page' containing brief information about local chapters of the Tuscan Association for the Blind and related Web site links. The users had to search for the telephone number and open the Web site of a given chapter.

7. Fill in a form.

This was a Web page with a form to fill in for sending comments, suggestions, or questions. The users had to fill in the form and send it.

The tasks carried out by users during the test B were:

1. Visit the page with books arranged by category.

This was a content page in which the information was organised by using 'heading levels' and local links to skip directly to a specific category. The user was asked to look for a specific piece of information (i.e. the price of a certain book of a given category).

2. Visit the Web page "Publisher shops".

This page was a simple content page in which 'heading levels' were used to logically group the different pieces of information. The user was asked to find the address of a given shop of the publishing house. 
3. Visit the alphabetically ordered list of authors who publish for the publishing house.

This information was in a content page in which both 'heading levels' and an index combo-box were used to organize page information. The user had to search for the number of authors having a name starting with a specific letter.

4. Read the biography of the author who wrote the third book available in the monthly book list.

In the home page, in addition to the links pointing to main Web site sections, some general information and events were available, among which, the monthly list of best sellers.

5. Users were asked to search for the interview with the author of the book of the month.

6. Filling in a form.

Users had to send a short message with some personal information (e.g., address, e-mail, and so on).

7. Searching within the Web site.

Every page had a search edit box by which users could search within the Web site. Users were asked to perform a search for a specific book.

Even if no specific task was assigned for each criterion, while navigating within the two Web sites, users could actually test all the applied criteria. For instance, "Location of the navigation bar" (or submenu) was tested in each Web page because two specific hidden labels were available - i.e. "Navigation bar:" and "Submenu:". Moreover, the criteria used to identify the main page content - i.e. the link 'skip to content' or the heading level 1 applied to the title of the page (not to be confused with <title> tag content) - could be "recognised" in each page when navigating freely or for carrying out the tasks. The same also occurred for other criteria such as "Page information" (e.g., path within the page title), "Addition of sounds" (e.g. differentiation of link types, or short sounds played at page loading), and so on.

While users performed the test, they were free to navigate as they pleased. A "Next task" button was available to start the following task when the user decided that the goal had been reached or decided to give up. The indication of the next task to accomplish appeared in a popup menu, which contained a "Continue the test" button to proceed with the test.

\section{RESULTS}

All data gathered through the testing procedure were analysed in order to evaluate the overall improvement of the Web site after the application of our criteria. Such improvement was measured in terms of navigation time saved by users in accomplishing given tasks. Log files created by the logging tool used for capturing data were analyzed. Both time and task accomplishment data were available thanks to those files for each test session (See section 3.2.3).

The difference between the time spent performing each task in Session ${ }_{(c)}$ and Session $_{(i)}$ (performed respectively in "control site" and "implemented site") was used to verify if and to what extent the application of our criteria had improved navigability. So, the time saved by users was taken as an indicator of Web site 
improvement. Moreover, the captured events stored in the log files pointed out that all tasks were practically accomplished by the users.

\subsection{STATISTICAL ANALYSIS}

The analysis was performed taking the users as the statistical unit and considering the navigation time (s) by i) all users $(n=40)$, ii) blind versus lowvision users $\left(n_{B L I N D}=24 ; n_{\text {LOW }}=16\right)$ and iii) group $A$ versus group $B\left(n_{A}=n_{B}=20\right)$.

The paired t-test was used to compare dependent samples (control vs. implemented site and tasks) while the t-test was used for independent samples (low vs. blind users). $\alpha=0.05$ was selected as the level of significance.

We found a highly significant reduction of the time (s) required to complete all tasks in the implemented site a) by all users (Figure 7; paired t-test: $n=40, d f=39$, $t=7.15, p<0.001)$ and $b)$ in the two groups (A and B) taken separately.

\section{ALL USERS}

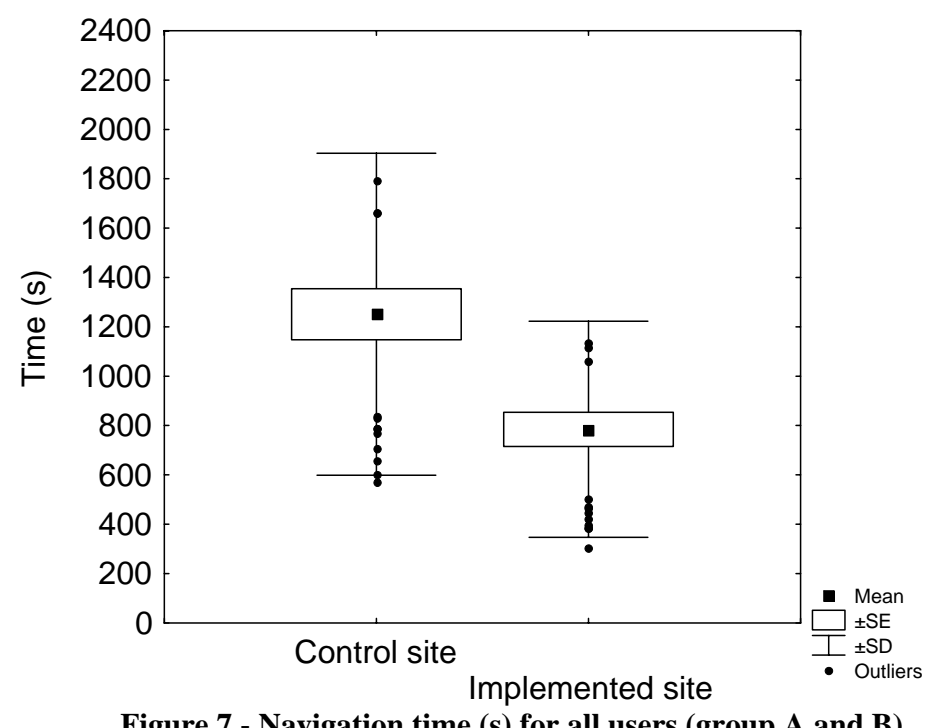

GROUP A

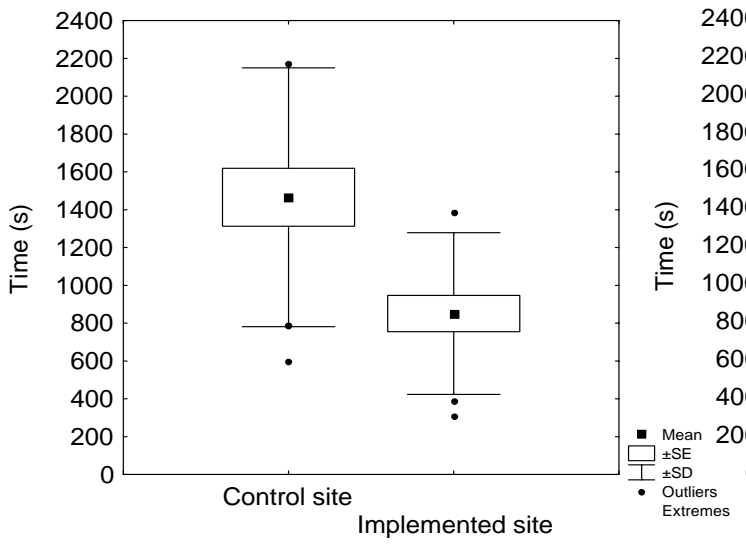

GROUP B

Figure 8 - Navigation time (s) for group A (left) and group B (right). 
Both blind users (Figure 9, left) and low-vision users (Figure 9, right) saved a highly significant amount of time when navigating in the implemented site (paired t-test: $\mathrm{n}_{\text {BLIND }}=24, \mathrm{df}=23, \mathrm{t}=5.23, \mathrm{p}<0.001 ; \mathrm{n}_{\mathrm{LOW}}=16, \mathrm{df}=15, \mathrm{t}=7.03, \mathrm{p}<0.001$ )

\section{BLIND USERS}

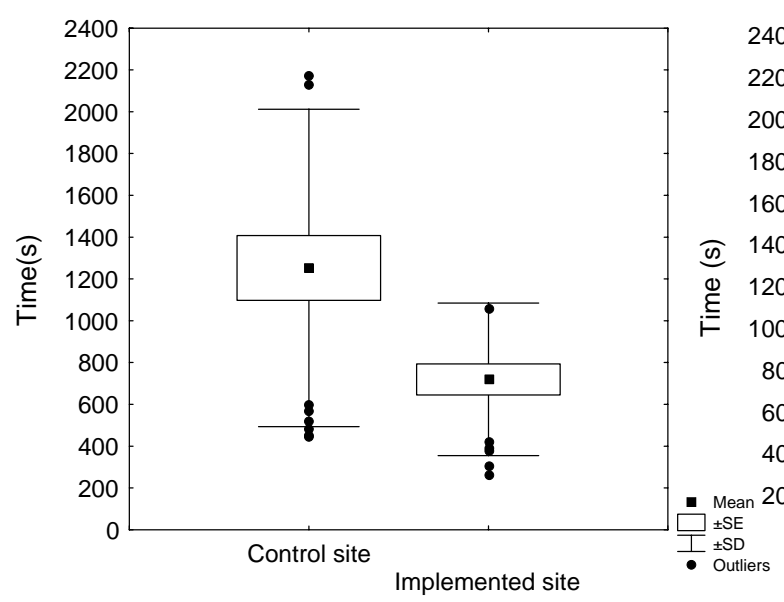

LOW VISION USERS

Figure 9 - Navigation time by blind users (left) and low-vision users (right).

On average, blind users saved more time than vision-impaired ones (about $42 \%$ and $29 \%$ respectively). However, the difference between blind and low-vision users, in terms of time saved, was not significant (t-test: $n_{B L I N D}=24, n_{L O W}=16$, $\mathrm{t}=1 . .25$, $\mathrm{df}=38$, ns). In fact, as mentioned in section 4.1 , the low-vision users included in our testing relied substantially on a screen reader for operating due to their serious visual deficit. Some visual features were used just to orientate within the page, for instance by colour changing. The high range of variability observed in the time saved by blind users (Figure 10) may explain this result. The time saved is calculated as the difference between the time required to navigate the control site and the navigation time needed for the imnlemented site

\section{IMPLEMENTED SITE:}

TIME SAVING

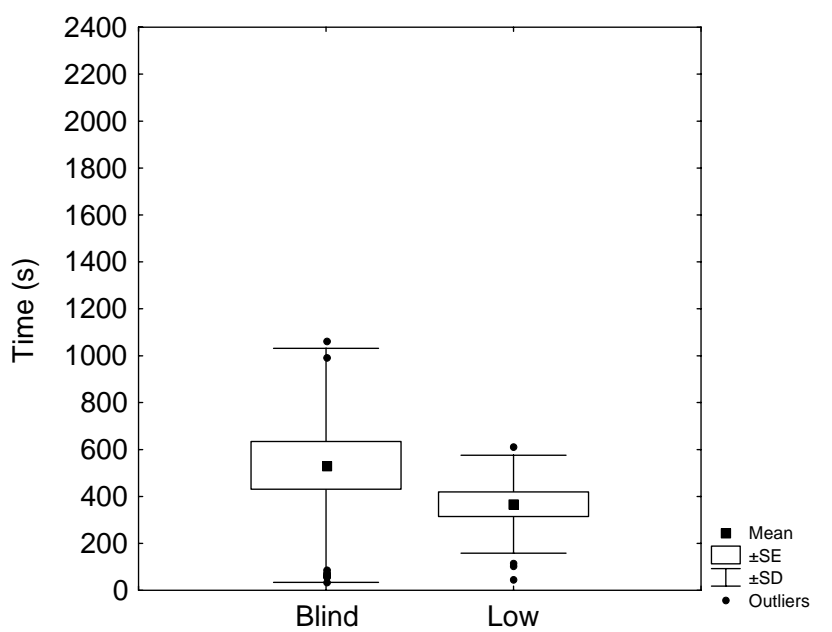

Figure 10 - Time comparison when navigating the implemented site. 
We found that the time saved by the users varied significantly among the different tasks both in group A (high significant, $\mathrm{p}<0.01$ ) and group $\mathrm{B}$ (significant, $\mathrm{p}<0.05)$ - repeated measures ANOVA: $\mathrm{n}_{\mathrm{A}}=20, \mathrm{df}=6, \mathrm{~F}=6.40, \mathrm{p}<0.01 ; \mathrm{n}_{\mathrm{B}}=20, \mathrm{df}=6$, $\mathrm{F}=3.45, \mathrm{p}<0.05)$.

GROUP A

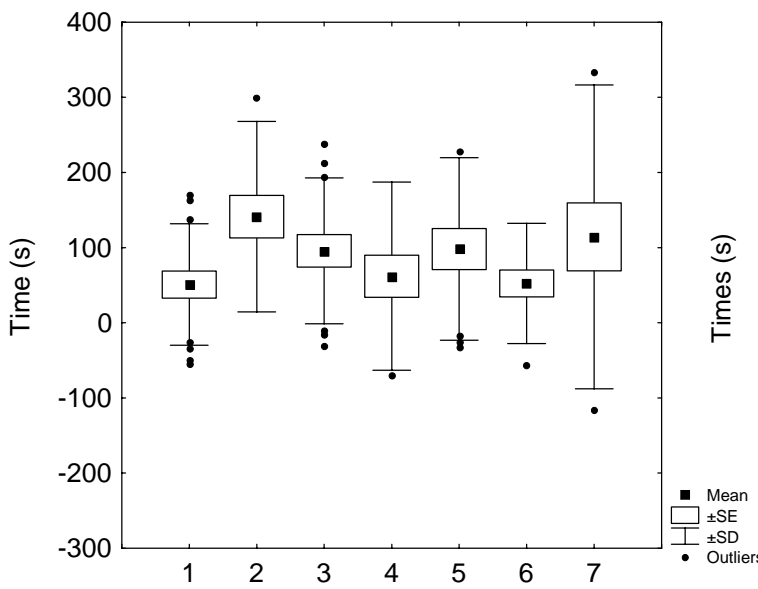

GROUP B

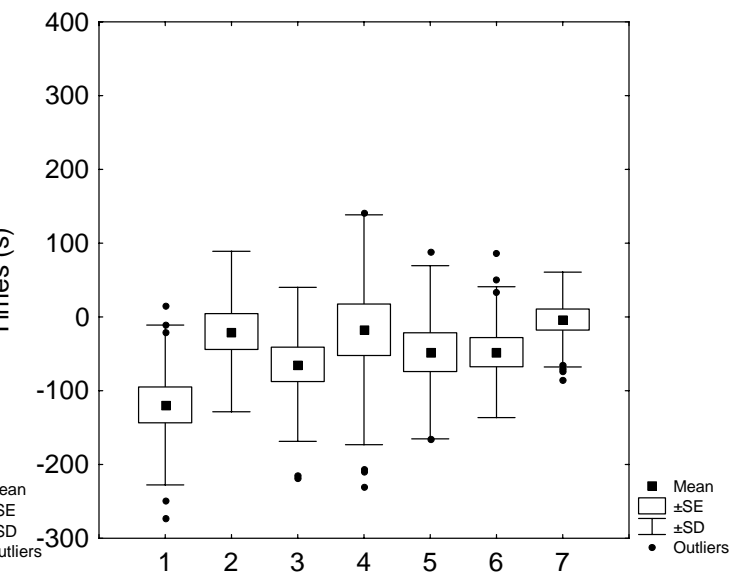

Figure 11 - Difference of time required to perform each task in control and implemented sites by users of group A (left) and group B (right).

\subsection{QUALITATIVE ANALYSIS}

Subjective information was gathered through a questionnaire filled in by all users. In particular, users were asked to express their opinions about sounds, colours, magnifiers, and so on.

$85 \%$ of the users considered the sound added to page loading to be helpful. However, $60 \%$ would also like to associate different sounds to distinct types of links (internal, external, local). All low-vision users found that the association of different colours to distinct areas helped spotting important sections in the page (e.g. area of application form, navigation bar, etc.). Colour contrast was considered unhelpful by $60 \%$ of low-vision users, who suggested using the combination white text/dark background, instead of the one used in the test site (black text/white background). About $2 / 3$ of all users used shortcuts and specific screen reader commands during the navigation.

Users were asked to write some remarks or differences observed between the two Web site versions. Almost all of the blind users judged particularly helpful (1) the application of headings to group information, (2) the usage of hidden labels for marking navigation bar and side submenu, and (3) the repartition of several files into various tables (one per category), obtained by using the "summary" attribute together with the name of each category. Low-vision users particularly appreciated magnification and colour change of background/text when the mouse cursor hovered over links.

Some blind users suggested the application of different sounds (1) to distinguish between failure and success (of a process); (2) to let the user know when they are writing in an edit box or when, in a field, there are no more characters to cancel 
using the backspace key. Low-vision users suggested better highlighting the separation among information blocks to facilitate the identification of each block.

\subsection{DISCUSSION}

Our results show that both blind and low-vision users benefited from the application of our criteria, saving on average around $37.29 \%$ of their navigation time $\left(42.93 \%\right.$ by Group ${ }_{\mathrm{A}}$ and $30.73 \%$ by Group $\left.{ }_{\mathrm{B}}\right)$. Time values showed that blind people saved more time than low-vision users ( $42.53 \%$ vs. $29.40 \%)$. Probably lowvision users can obtain some benefit from the use of magnifying programs even in the sites where our criteria are not applied. However, the difference in the time saved by low-vision and blind users is not statistically significant, as expected by the fact that i) our criteria are mainly targeted to screen reader navigation and ii) the low-vision users involved in the experiment can be considered almost blind. So, the low-vision and blind users can be considered a homogenous group.

The wide range of time difference shown and Figure 11 is possibly due to differences in task difficulty. Task $1(34.73 \%)$ and $6(32.45 \%)$ in Test $^{\mathrm{A}}$ and Task 4 $(9.30 \%)$ and $7(3.43 \%)$ in Test $^{\mathrm{B}}$ were the least influenced by our criteria, probably because the main criterion involved in these two tasks was the application of heading levels, which are not so crucial for low-vision users, unless the content of the page includes a very high number of links and/or is overloaded by confusing information. All these tasks consisted of searching for information within the home page or a first level page, which had few links and were not very long, thus making them relatively simple. Task $2^{\mathrm{A}}$ (i.e. looking for information in a long page) turned out to be the most influenced by our criteria, probably due the fact that low-vision users could considerably reduce their navigation by using side submenus (e.g. local links or list boxes) to move quickly to a specific section of the page. In Test ${ }^{\mathrm{B}}$, Tasks 1 and 3 (i.e., looking for information in a long page as well) benefited from the greatest savings: $55.86 \%$ and $49.14 \%$ respectively. The criteria affecting mainly these tasks are "Proper link content" and "Logical partition elements". Especially in Site $^{B}{ }_{(c)}$ links had inappropriate content. In fact, the alternative descriptions for the graphical links "Titles", "Authors", "Categories" and so on, were meaningless: for example "Link1", "Link2", etc.. Such link content was accessible - i.e. recognisable by the screen reader - but not usable: users had to open each linked page in order to find out whether it was the desired page or not. Since all links of Site $_{(\text {i) }}{ }_{\text {i }}$ had more appropriate content (e.g., Titles, Authors, Categories, etc.), we expected a significant time saving in performing tasks on the "implemented" site ${ }^{\mathrm{B}}$ due to clearer link contents. However, this was not the case because users used a screen reader command for reading the link address before opening the page in site

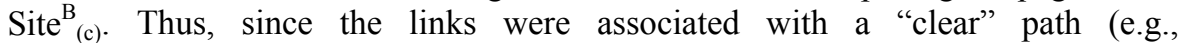
http://.../authors.html), users were able to recognize the content of the corresponding page quite easily. In practice, users resorted to a certain "trick" to overcome the difficulties encountered in understanding link labels. Consequently the time saving is less significant and sometimes it is even negative. From this experience, we learnt that when considering a Web site for carrying out a test to evaluate the "proper link content" criterion, it is important not to give a selfexplaining link address.

Concluding, on average, the two tests revealed that the application of our criteria to the Web sites led to a significant time saving for all users and tasks. 


\section{CONCLUSIONS}

In this paper, we first introduce a set of usability criteria identified to improve Web navigation for vision-impaired people, and then we present the empirical results of a user testing designed to evaluate the concrete impact of such criteria on the Web interface. To this end, two testing were conducted by involving forty visually-impaired users. In practise, we report on an experiment we conducted to validate the utility of the 15 identified criteria.

The protocol used for the user testing, involving users with total/almost total visual deficit, based on remote evaluation techniques. Two versions of two Web sites were considered: a "control version" without criteria and an "implemented version" specially designed/modified by applying our usability criteria. A questionnaire was used to collect information on navigation quality from users. User testing and questionnaire results reveal that our usability criteria improved Web site navigability both in quantitative and qualitative terms, by reducing the navigation time of about $37 \%$ on average and by making the Web site more friendly to visionimpaired users. By analysing the objective data (time required for each task) we can observe that there is a significant reduction in the time spent by users performing the assigned tasks. Furthermore, by subjective data collected through the questionnaires we observed that the navigation was more suitable and satisfactory in the Web sites including our criteria. So, combining the two kinds of information we can conclude that when the criteria are applied to Web sites, the Web interaction of visually-impaired users is influenced positively in terms of efficiency and satisfaction as well. Thus, the usability of Web sites is improved for this special category of users.

The guidelines tested with the two experiments conducted refer mainly to Web desktop interfaces. However, we believe that these criteria can be a good basis for identifying an appropriate set of accessibility and usability guidelines also for mobile navigation and future work is planned to investigate this possibility. In any case, the criteria we identified can help developers to become aware of the main aspects affecting Web navigation for visually-impaired users. A possible inconvenience could be related to the effort required of developers to work with guidelines: applying or evaluating them could take long time. To this end, we are currently working on the development of a tool that should be able to automatically check whether the design criteria proposed are followed in the Web site considered in order to facilitate their application and support designers right from the development phase. The tool should also support multiple guidelines without requiring modifications in its internal implementation, including guidelines specified directly by the designer.

\section{ACKNOWLEDGMENTS}

The authors wish to thank to Ivan Norscia for the help to improve the paper presentation, Marzia Bandoni for her contribution in one of the two tests, and all people who participated in the user testing.

\section{REFERENCES}

Abascal J., Arrue M., Fajardo I., Garay N., Tomás J., 2003. Use of Guidelines to automatically verify Web accessibility. Universal Access in the Information Society. Special Issue on "Guidelines, standards, methods and processes for software accessibility" (Guest editors: Gulliksen J., Harker S., Vanderheiden G.). Springer Verlag, 2003. 
Barnicle, K., 2000. Usability Testing with Screen Reading Technology in a Windows Environment. Proceedings of the 2000 Conference on Universal Usability (CUU-00), pp. 102-109, ACM Press, November 16-17 2000.

Bobby: Free Public Service for Web Accessibility evaluation by CAST (Center for Applied Special Technology) accessible at http://webxact.watchfire.com/.

Brewster, S.A., 1998. Using earcons to improve the usability of a graphics package. In Proceedings of BCS HCI'98, (Sheffield, UK), Springer-Verlag, pp 287-302.

Donker H., Klante P., Gorny P., 2002. The Design of Auditory User Interfaces for Blind Users. In: Proceedings of the Second NordiCHI, Tradition and Transcendence, Edited by O. W. Bertelsen, S. Boedker and K. Kuutti, October 19-23, 2002, Aarhus Denmark. New York 2002 : ACM Press pp. 149155.

Fukuda K., Saito S., Takagi H. and Asakawa C., Proposing New Metrics to Evaluate Web Usability for the Blind. In the proceedings ACM CHI'05 extended abstracts on Human factors in computing systems, April 02-07, 2005, Portland, OR, USA.

Goble C., Harper S., Stevens R., The Travails of Visually-impaired Web Travellers, Proceedings ACM Hypertext 2000, ACM Press.

Hanson V.L., Brezin J.P., Crayne S., Keates S., Kjeldsen R., Richards J.T., Swart C., Trewin S., Improving Web accessibility through an enhanced open-source browser, IBM Systems Journal 2005, pp.573-588.

Jaws for Windows, 2005. Freedom Scientific at http://www.freedomscientific.com.

Lee A., Hanson V., Enhancing Web Accessibility, Proc. ACM Multimedia 2003, pp.456-457, ACM Press.

Leporini, B., Criteria to improve Web site usability and accessibility when interacting through screen readers: definition, application, and evaluation. $\mathrm{PhD}$ thesis, Dipartimento di Informatica, Università di Pisa, September 2003.

Leporini, B., Paternò, F., 2004. Increasing Usability when Interacting through Screen Readers, International Journal Universal Access in the Information Society (UAIS), Springer Verlag, Vol.3, N.1.

Marion C.. Make way for Interactive Assistance, 2000, available at http://mysite.verizon.net/resnx4g7/7/MakeWay/MakeWay.htm

Mayhew D. J.. Principles and guidelines in software and user interface design. Prentice Hall, Englewood Cliffs, New Jersey, 1992.

Mankoff J., Fait H., Tran T., 2005. Is Your Web Page Accessible? A Comparative Study of Methods for Assessing Web Page Accessibility for the Blind, in proceedings ACM CHI'05, pp.41-50.

Nicolle, C., Abascal J., 2001. Inclusive design guidelines for HCI, p. 285, Taylor \& Francis, 2001.

Paganelli, L., Paternò, F., 2003. Tools for Remote Usability Evaluation of Web Applications through Browser Logs and Task Models, Behavior Research Methods, Instruments, and Computers, The Psychonomic Society Publications, 2003, 35 (3), pp.369-378, August 2003.

Scapin, D.L., Leulier, C., Vanderdonckt, J., Mariage, C., Bastien, Ch., Farenc, Ch., Palanque, Ph., and Bastide, R., 2000. A Framework for Organizing Web Usability Guidelines, in Ph. Kortum and E. Kudzinger (eds.), Proc. of 6th Conf. on Human Factors and the Web HFWeb'2000, Austin, 2000, accessible at http://www.tri.sbc.com/hfweb/scapin/Scapin.html .

Section 508 standards available at http://www.section508.gov/

Smith S. L. and Mosier J. N.. Guidelines for designing user interface software. Mitre Corporation Report MTR-9420, Mitre Corporation, 1986.

Stephanidis, C., Akoumianakis, D., Sfyrakis, M., \& Paramythis, A., 1998. Universal accessibility in HCI: Process-oriented design guidelines and tool requirements. In C. Stephanidis \& A. Waern (Eds.), Proceedings of the 4th ERCIM Workshop on "User Interfaces for All", Stockholm, Sweden, 19-21 October .

Takagi, H., Asakawa, C., Fukuda, K., and Maeda, J. 2004. Accessibility designer: visualizing usability for the blind. In Proceedings of the 6th international ACM SIGACCESS Conference on Computers and Accessibility (Atlanta, GA, USA, October 18 - 20, 2004). Assets '04. ACM Press, New York, NY, 177-184.

Theofanos, M.F., Redish, J., 2003. Bridging the gap: between accessibility and usability. ACM Interactions magazine, New York: ACM Press, Nov.-Dec. 2003 issue, pp.36-51

WAI Accessibility Guidelines. Web Accessibility Initiative, World Wide Web Consortium, 1999. Accessible at http://www.w3.org/wai

Web Content Accessibility Guidelines 2.0, W3C Working Draft 1 March 2004 available at http://www.w3.org/WAI/GL/WCAG20/ 